\title{
Drivers and Barriers to the Use of Health Information Exchange Amongst Clinicians in the Emergency Department
}

Ben Richter ${ }^{1}$, Brian Dixon ${ }^{2}$

${ }^{1}$ Indiana University School of Medicine, ${ }^{2}$ Indiana University Richard M. Fairbanks School of Public Health, Department of Epidemiology; Regenstrief Institute, Center for Biomedical Informatics

\section{Background:}

Health Information Exchange (HIE) describes the exchange of medical data between various health care organizations. Though research is limited, widespread use of HIE may improve patient outcomes while improving efficiency and thus lowering health care costs for patients. The paucity of existing research necessitates further study into the effects of HIE use in the clinical setting. The Indiana Network for Patient Care (INPC) is one of the most comprehensive HIE networks in the country, and provides an ideal environment for conducting research regarding factors that influence HIE use.

\section{Methods:}

A group of 20 clinicians from the Emergency Department were chosen to answer a set of questions regarding their HIE use. This group included physicians, nurse practitioners, physician assistants, and registered nurses from various health care organizations across the state of Indiana. Interview questions were centered around four main themes: Performance Expectancy, Effort Expectancy, Social Influence, and Facilitating Conditions. Interviews were recorded and transcribed, then subject to qualitative analysis using NVivo software.

\section{Results:}

The Single Sign-On and EHR Button were the most commonly discussed features in terms of facilitating HIE use. Providers used HIE most often when the patient reported previous admission at a different hospital, or when the patient was incapacitated and could not provide information. Although clinicians had unanimous social support for using HIE, inadequate training regarding HIE was apparent, and served as the most common barrier to its use.

Conclusion/Impact:

The implementation of Single Sign-On and access to the INPC via a button integrated into the user's EHR are critical for widespread use of HIE, while lack of physician training serves as a major barrier to its use. Implementing SSO and EHR button features while improving HIE training may spurn additional use of HIE and thus lower costs for both hospitals and patients. 